interacting. Now, Markus Müller at the Abdus Salam International Center for Theoretical Physics in Trieste, Italy and co-workers have shown that electrons in graphene do in fact interact strongly, and behave like a 'perfect fluid'.

A perfect fluid does not conduct heat and has zero viscosity, so it cannot exert stress on surrounding objects. Previously, researchers believed that electrons in graphene were an example of a Fermi liquid, meaning that there are no interactions between particles at low temperatures. This is true for electrons in metals, where electrons block the interactions between one another.

Müller, along with co-workers Jörg Schmalian at Iowa State University and Lars Fritz at Harvard University, used quantum kinetic theory to show that electrons in graphene have a very small ratio of viscosity (thickness) to entropy (disorder). This means that the electrons do interact strongly, and form a 'quantum critical' perfect fluid, which has only been seen in a quark-gluon plasma created in the Relativistic Heavy Ion Collider.

The findings imply that graphene sheets could show interesting quantum phenomena such as electronic turbulence. This could be important for applications of graphene in nanoscale electronics.

\section{MIM RESONATORS}

\section{Pixel perfect}

Nano Lett. 9, 2579-2583 (2009)

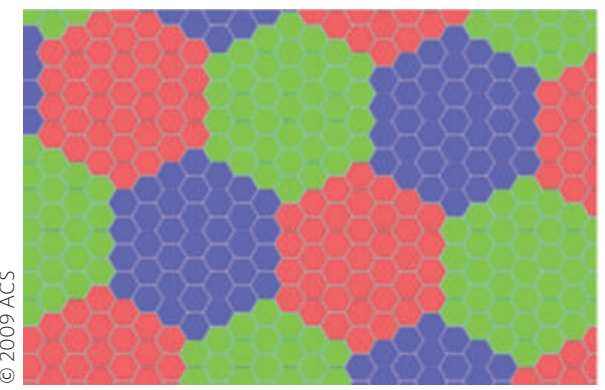

Colour filters are essential for separating red, green and blue wavelengths of light in digital cameras and displays. Now, Kenneth Diest and colleagues at the California Institute of Technology in Pasadena have described a tiny new type of colour filter that could provide the highest screen resolution so far.

Their technology is based on metalinsulator-metal (MIM) resonators, in which a very thin layer of insulating material is sandwiched between two metal layers. Light passing through the insulating layer interferes with itself, so only certain colours get through.
Diest and co-workers built MIM resonators with silver nitride as the insulating layer. They found that each device transmitted different wavelengths depending on the shape and size of the resonator. Furthermore, the researchers performed simulations of a MIM resonator containing lithium niobate as the insulating layer. They found that they could tune the output colour from the device simply by applying an electric field between the metal plates.

The researchers propose building a 'MIM pixel' by sticking together three different MIM resonators tuned to transmit red, green and blue light. The MIM pixel would be between 3 and $5 \mu \mathrm{m}$ in size, which is far smaller than pixels from the highestdefinition colour filters in use today.

\section{NANOWIRES}

\section{Plug and play}

Appl. Phys. Lett. 94, 263110 (2009)

Plug-and-socket-based electrical connections are a traditional means of delivering power to electronic circuits. However, despite the dramatic downsizing of electronic devices, such conventional electrical connectors cannot be scaled down to submillimetre regimes because the mechanisms for making the electrical and physical connections are not the same. Now, Ali Javey and colleagues at the University of California at Berkeley have devised electrical connectors from hybrid core-multishell nanowire forests in which the electrical and physical contact is made using the same active area.

The nanowire connectors consisted of germanium nanowire cores grown on silicon substrates, covered with a 50-300-nm-thick layer of parylene - a hydrophobic polymer - and finally a 45-nm-thick silver shell. The germanium nanowires were several micrometres in length with a density of 10-20 nanowires per micrometre at the substrate surface and 1-2 nanowires per micrometre at the top of the nanowires. The parylene acts to enhance adhesion between interpenetrating nanowire arrays, and the silver shell is necessary for electrical connect.

The nanowire electrical connectors were successfully used to connect a lightemitting-diode array to a wall-mounted battery array.

The definitive versions of these Research Highlights first appeared on the Nature Nanotechnology website, along with other articles that will not appear in print. If citing these articles, please refer to the web version.

\section{Top down Bottom up}

\section{East meets northwest}

Nanodiamonds allow insoluble drugs to be dispersed in water.

Dean Ho had been interested in therapeutic drug delivery for some time before a meeting with Eiji Osawa took his research in a new direction. Ho, an assistant professor in the department of biomedical and mechanical engineering at Northwestern University, had experience with cancer therapies; Osawa, who worked for NanoCarbon Research, a private research institute in Nagano, Japan, was an expert on fullerenes and nanocarbons. Osawa had, in fact, proposed that $\mathrm{C}_{60}$ could be a stable form of carbon in 1970 15 years before its discovery was published in Nature. Following their interactions with Osawa, $\mathrm{Ho}$ and colleagues realized the potential that nanodiamonds had to advance their own research.

Many drugs show promising effects but cannot be delivered effectively because they are insoluble in water. Nanodiamonds, however, can be readily suspended in water when they are functionalized with carboxyl groups. Moreover, Ho and co-workers showed that nanodiamonds can aid the dispersal in water of water-insoluble drugs, making them viable therapeutic candidates (ACS Nano doi:10.1021/nn900480m; 2009). Three water-insoluble anticancer and anti-inflammatory drugs were shown to physisorb onto nanodiamond surfaces and disperse in water, without a reduction in function. The resulting dispersion showed a small aggregate size, improving the chances of the drug entering cells.

Ho enjoyed witnessing the "clear evolution of each researcher towards an appreciation of the challenges that faced each of their colleagues". The corresponding challenge was maintaining a tight focus: "a large number of researchers can sometimes result in diverging objectives". According to $\mathrm{Ho}$, overcoming this obstacle involves both formulating objectives that unify the disciplines involved, and communication that begins at the earliest stages: "recognition from the outset that these challenges can be overcome with interaction and dialogue is key." 